Proceedings of

The 37Th TANiguchi Symposium on

TOPOLOGY AND TEICHMÜLlER SPACES

held in Finland, July 1995

ed. by Sadayoshi KOJIMA et al.

(c) 1996 World Scientific Publishing Co.

pp. 253-264

\title{
CHARACTER VARIETY OF REPRESENTATIONS OF A FINITELY GENERATED GROUP IN $\mathrm{SL}_{2}$
}

\author{
KYOJI SAITO
}

(Received January 10, 1996)

This is a partial exposition of [S4, S5, S6], which study the space of representations of a (finitely generated) group $\Gamma$ in $\mathrm{SL}_{2}$ and $\mathrm{GL}_{2}$ in an attempt of its application in geometry: Teichmüller spaces, knot theory, hyperbolic manifolds, moduli spaces, $\cdots$, etc. (see for instance, $[\mathrm{A}],[\mathrm{Be}],[\mathrm{Br}],[\mathrm{C}-\mathrm{C}-\mathrm{G}-\mathrm{L}-\mathrm{S}],[\mathrm{C}-\mathrm{S}],[\mathrm{F}-\mathrm{K}],[\mathrm{G}],[\mathrm{H}],[\mathrm{H}-\mathrm{L}-\mathrm{M}]$, $[\mathrm{H}-\mathrm{K}],[\mathrm{J}-\mathrm{W}],[\mathrm{K}],[\mathrm{Kj}],[\mathrm{Ko}],[\mathrm{Kr}],[\mathrm{Ma}],[\mathrm{Mu}],[\mathrm{N}-\mathrm{Z}],[\mathrm{O}],[\mathrm{S}],[\mathrm{S}-\mathrm{S}],[\mathrm{T}],[\mathrm{W}],[\mathrm{We}]$, $[\mathrm{Wo}],[\mathrm{Y}], \cdots$, etc). For simplicity, we omit the case for $\mathrm{GL}_{2}$ in the present exposition. Let us explain the main result of the present paper.

Let $\Gamma$ be a group. The purpose of the present paper is to introduce the character variety $\mathrm{Ch}\left(\Gamma, \mathrm{SL}_{2}\right)$ in order to parameterize conjugacy classes of representations of $\Gamma$ in $\mathrm{SL}_{2}$ in a functorial way. At first, the character variety is introduced as a scheme over $\mathbb{Z}$, independent of the coefficient ring of representations in question. Then, the scaler field is specialized to $\mathbb{R}$ to obtain results on representations in $\mathrm{SL}_{2}(\mathbb{R})$ and in $\mathrm{SU}(2)$ with respect to the classical topology. Let us explain this briefly.

Let $\operatorname{Hom}\left(\Gamma, \mathrm{SL}_{n}\right)$ be the functor $R \in\{$ commutative rings with 1$\} \mapsto \operatorname{Hom}(\Gamma$, $\left.\mathrm{SL}_{n}(R)\right) \in\{$ sets $\}$. The functor is representable (see $\S 1.3$ Lemma) and so, for an abuse of notation, we denote by the same $\operatorname{Hom}\left(\Gamma, \mathrm{SL}_{n}\right)$ the scheme over $\mathbb{Z}$ representing the functor. The group scheme $\mathrm{PGL}_{n}$ acts on $\operatorname{Hom}\left(\Gamma, \mathrm{SL}_{n}\right)$. Whether the universal categorical quotient $\operatorname{Hom}\left(\Gamma, \mathrm{SL}_{n}\right) / / \mathrm{PGL}_{n}$ (Mumford [Mu1]) defined over $\mathbb{Z}$ exists or not seems to be a hard and unsolved question. Instead of asking directly for the quotient space, we introduce $i)$ the character variety $\mathrm{Ch}\left(\Gamma, \mathrm{SL}_{2}\right)$ together with its discriminant subvariety $D_{\Gamma}$ as schemes over $\mathbb{Z}$ abstractly, and $i i$ ) the $\mathrm{PGL}_{2}$-invariant morphism $\pi_{\Gamma}: \operatorname{Hom}\left(\Gamma, \mathrm{SL}_{2}\right) \rightarrow \mathrm{Ch}\left(\Gamma, \mathrm{SL}_{2}\right)$, for which we prove that i) the restriction of $\pi_{\Gamma}$ on the complement of $\pi_{\Gamma}^{-1}\left(D_{\Gamma}\right)$ is a principal $\mathrm{PGL}_{2}$-bundle with respect to the etal topology, and $i i)$ the inverse image $\pi_{\Gamma}^{-1}\left(D_{\Gamma}\right)$ is a subfunctor of $\operatorname{Hom}\left(\Gamma, \mathrm{SL}_{2}\right)$ consisting of abelian or reducible representations. This implies that the complement $\operatorname{Hom}^{*}\left(\Gamma, \mathrm{SL}_{2}\right):=\operatorname{Hom}\left(\Gamma, \mathrm{SL}_{2}\right) \backslash \pi^{-1}\left(D_{\Gamma}\right)$ consists of absolutely irreducible representations, and that $\operatorname{Hom}^{*}\left(\Gamma, \mathrm{SL}_{2}\right)$ has the universal categorical quotient space $\mathrm{Ch}^{*}\left(\Gamma, \mathrm{SL}_{2}\right):=\operatorname{Ch}\left(\Gamma, \mathrm{SL}_{2}\right) \backslash D_{\Gamma}$ defined over $\mathbb{Z}$. Then the result is specialized 
to $\mathbb{R}$-coefficient. Namely, the complement $\mathrm{Ch}^{*}\left(\Gamma, \mathrm{SL}_{2}\right)(\mathbb{R}):=\operatorname{Ch}\left(\Gamma, \mathrm{SL}_{2}\right)(\mathbb{R}) \backslash D_{\Gamma}(\mathbb{R})$ of the real discriminant decomposes into a disjoint union of two semialgebraic open components $H_{\Gamma}(\mathbb{R})$ and $T_{\Gamma}(\mathbb{R})$ such that i) $\operatorname{Hom}^{*}\left(\Gamma, \mathrm{SL}_{2}\right)(\mathbb{R})$ is a principal $\mathrm{PGL}_{2}(\mathbb{R})$ bundle over $H_{\Gamma}(\mathbb{R})$, and ii) $\operatorname{Hom}^{*}\left(\Gamma, \mathrm{SU}_{2}(\mathbb{C})\right)$ is a principal $\mathrm{PU}(2)$ bundle over $T_{\Gamma}(\mathbb{R})$, respectively. This fact has an application: the Teichmüller space $\mathcal{T}_{g, n}$ carries a natural semi-algebraic structure defined over $\mathbb{Z}[\mathrm{S} 5]$.

The $\S 1$ prepares notations of a representation variety $\operatorname{Hom}\left(\Gamma, \mathrm{SL}_{2}\right)$ defined over $\mathbb{Z}$. The $\S 2$ studies the $\mathrm{PGL}_{2}$-invariants of $M_{2} \times M_{2}$ as the building block. The universal character ring $R\left(\Gamma, \mathrm{SL}_{2}\right)$ is introduced in $\S 3$, and we put $\mathrm{Ch}\left(\Gamma, \mathrm{SL}_{2}\right):=$ $\operatorname{Spec}\left(R\left(\Gamma, \mathrm{SL}_{2}\right)\right)$. The principal $\mathrm{PGL}_{2}$-bundle structure on $\operatorname{Hom}^{*}\left(\Gamma, \mathrm{SL}_{2}\right)$ over $\mathrm{Ch}^{*}(\Gamma$, $\left.S L_{2}\right)$ with respect to the etal topology is formulated in $\S 4$ Theorem A, and that with respect to the classical topology is formulated in $\S 5$ Theorem C.

\section{$\S 1$ Universal representation of a group $\Gamma$ in $\mathrm{SL}_{n}$}

This section is devoted for a preparation of notion and terminology for the representation varieties. One is referred to $[\operatorname{Pr} 1, \operatorname{Pr} 2][\mathrm{L}-\mathrm{M}]$ etc.

1.1. Let $\Gamma$ be a group. As in the introduction, for a fixed $n \in \mathbb{Z}_{>0}, \operatorname{Hom}\left(\Gamma, \mathrm{SL}_{n}\right)$ is the functor: $R \in\{$ commutative rings with 1$\} \mapsto \operatorname{Hom}\left(\Gamma, \mathrm{SL}_{n}(R)\right) \in\{$ sets $\}$. In order to fix the notation for the present paper, we state precisely the representability of the functor in the next lemma.

Lemma (the representability of $\operatorname{Hom}\left(\Gamma, \mathrm{SL}_{n}\right)$ ).

(1) For the given $\Gamma$ and $n \geq 1$, there exists a pair $\left(A\left(\Gamma, \mathrm{SL}_{n}\right), \sigma\right)$ of a commutative ring $A\left(\Gamma, \mathrm{SL}_{n}\right)$ with 1 and representation $\sigma: \Gamma \rightarrow \mathrm{SL}_{n}\left(A\left(\Gamma, \mathrm{SL}_{n}\right)\right)$ such that for any commutative ring $R$ with 1 , the correspondence:

$$
\varphi \in \operatorname{Hom}^{r i n g}\left(A\left(\Gamma, \mathrm{SL}_{n}\right), R\right) \mapsto \varphi \circ \sigma \in \operatorname{Hom}^{g r}\left(\Gamma, \mathrm{SL}_{n}(R)\right)
$$

is a bijection.

(2) The pair $\left(A\left(\Gamma, \mathrm{SL}_{n}\right), \sigma\right)$ is unique up to an isomorphism of the ring $A\left(\Gamma, \mathrm{SL}_{n}\right)$ which commutes with the universal representation $\sigma$.

(3) If $\Gamma$ is a finitely generated group, then $A\left(\Gamma, \mathrm{SL}_{n}\right)$ is a finitely generated ring over $\mathbb{Z}$ and hence is noetherian.

The lemma is proven by routine arguments. Here we give an explicit description of $\left(A\left(\Gamma, \mathrm{SL}_{n}\right), \sigma\right)$ without a proof. For each $\gamma \in \Gamma$, consider a $n \times n$ matrix:

$$
\sigma(\gamma):=\left(a_{i j}(\gamma)\right)_{i, j=1, \cdots, n}
$$

Then $A\left(\Gamma, \mathrm{SL}_{n}\right)$, called the universal representation algebra, is generated by the entries $a_{i j}(\Gamma) i, j=1, \cdots, n \gamma \in \Gamma$, and divided by the ideal generated by all entries of the matrices $\sigma(e)-I_{n}\left(I_{n}=\right.$ the $n \times n$ unit matrix $)$ and $\sigma(\gamma \delta)-\sigma(\gamma) \sigma(\delta)$ and $\operatorname{det}(\sigma(\gamma))-1$.

$$
A\left(\Gamma, \mathrm{SL}_{n}\right):=\mathbb{Z}\left[a_{i j}(\gamma) \text { for } \gamma \in \Gamma \text { and } 1 \leq i, j \leq n\right] / I
$$


where

$$
\begin{aligned}
& I:=\left(a_{i j}(e)-\delta_{i j}, a_{i j}(\gamma \delta)-\sum_{k} a_{i k}(\gamma) a_{k j}(\delta), \operatorname{det}(\sigma(\gamma))-1\right. \\
& \quad \text { for } 1 \leq i, j \leq n \text { and } \gamma, \delta \in \Gamma) .
\end{aligned}
$$

By definition, the map $\sigma: \gamma \in \Gamma \rightarrow \sigma(\gamma) \in \mathrm{SL}_{n}\left(A\left(\Gamma, \mathrm{SL}_{n}\right)\right)$ is a representation of $\Gamma$, which is called the universal representation of $\Gamma$ in $\mathrm{SL}_{n}$.

1.2. Let $\mathrm{PGL}_{n}$ be the group scheme ([SGAIII]), whose coordinate ring $A\left(\mathrm{PGL}_{n}\right)$ is given by the subring $A_{0}\left(\mathrm{GL}_{n}\right)$ of the coordinate ring $A\left(\mathrm{GL}_{n}\right):=\mathbb{Z}\left[x_{i j} 1 \leq i, j \leq\right.$ $n]_{\operatorname{det}(X)}$ (here $X:=\left(x_{i j}\right)_{i, j=1}^{n}$ ) of $\mathrm{GL}_{n}$ consisting of homogeneous elements of degree 0 . The adjoint action of $\mathrm{PGL}_{n}$ on $\operatorname{Hom}\left(\Gamma, \mathrm{SL}_{n}\right)$ is written in terms of its dual action:

$$
\mathrm{Ad}: A\left(\Gamma, \mathrm{SL}_{n}\right) \rightarrow A\left(\Gamma, \mathrm{SL}_{n}\right) \otimes_{\mathbb{Z}} A\left(\mathrm{PGL}_{n}\right),
$$

sending an entry of $\sigma(\gamma)$ to the same entry of $X^{-1} \sigma(\gamma) X$. Obviously, the coefficients of characteristic polynomial of $\sigma(\gamma)$ for $\gamma \in \Gamma$ are invariants under the adjoint action.

1.3. ¿From now on, we switch to the case $n=2$. Let us list up some relations among the $\mathrm{PGL}_{2}$-invariants $\operatorname{tr}(\sigma(\gamma))$ for $\gamma \in \Gamma$. The first one is trivial:

$$
\operatorname{tr}(\sigma(e))=2 .
$$

The next one follows from the Cayley-Hamilton relation: $\sigma(\gamma)^{2}+\operatorname{det}(\sigma(\gamma)) \cdot I_{2}=$ $\operatorname{tr}(\sigma(\gamma)) \cdot \sigma(\gamma)$. Multiply $\sigma\left(\gamma^{-1} \delta\right)$ from right and take traces. So we obtain:

$$
\operatorname{tr}(\sigma(\gamma \delta))+\operatorname{tr}\left(\sigma\left(\gamma^{-1} \delta\right)\right)=\operatorname{tr}(\sigma(\gamma)) \cdot \operatorname{tr}(\sigma(\delta))
$$

for $\gamma$ and $\delta \in \Gamma$ (cf. [F-K, formulas (2), pp.338]).

\section{$\S 2 . \mathrm{PGL}_{2}$-invariants for pairs of $2 \times 2$ matrices}

We study the invariants of the diagonal adjoint action of $\mathrm{PGL}_{2}$ on the space $M_{2} \times M_{2}$ of pair $(A, B)$ of $2 \times 2$ matrices. The morphism $\tilde{\pi}: M_{2} \times M_{2} \rightarrow \mathbb{A}^{5}$ given by $(A, B) \mapsto$ $(\operatorname{tr}(A), \operatorname{tr}(B), \operatorname{tr}(A B), \operatorname{det}(A), \operatorname{det}(B))$ is shown to be the universal quotient map $(\S 2.3$ Lemma A,B). The discriminant $\Delta$ for $\tilde{\pi}$ is introduced ( $\$ 2.4$ Lemma $C$ ) so that the $\tilde{\pi}$ is a principal $\mathrm{PGL}_{2}$-bundle on the complement of the discriminant loci $\Delta=0(\S 2.5$ Lemma D). These are building blocks of character variety defined over $\mathbb{Z}$.

2.1. Let $M_{2} \times M_{2}$ be the space of pairs of $2 \times 2$ matrices. The $X \in \mathrm{PGL}_{2}$ acts on $(A, B) \in M_{2} \times M_{2}$ from the right diagonally by letting $(A, B) \cdot \operatorname{Ad}(X):=$ $\left(X^{-1} A X, X^{-1} B X\right)$. So we have the dual action on the coordinate ring:

$$
\mathrm{Ad}: \mathbb{Z}\left[M_{2} \times M_{2}\right] \rightarrow \mathbb{Z}\left[M_{2} \times M_{2}\right] \otimes_{\mathbb{Z}} A\left(\mathrm{PGL}_{2}\right)
$$

sending an entry of $(A, B)$ to the corresponding entry of $(A, B) \cdot \operatorname{Ad}(X)$ where $\mathbb{Z}\left[M_{2} \times\right.$ $\left.M_{2}\right]$ is the polynomial ring generated by 8 entries of $(A, B)$. 
2.2. Let us consider the morphism

$$
\tilde{\pi}: M_{2} \times M_{2} \rightarrow \mathbb{A}^{5}:=\operatorname{Spec}(\mathbb{Z}[\underline{T}, \underline{D}]),
$$

where $\mathbb{Z}[\underline{T}, \underline{D}]$ denotes the polynomial ring $\mathbb{Z}\left[T_{1}, T_{2}, T_{3}, D_{1}, D_{2}\right]$ of the 5 indeterminates and $\tilde{\pi}$ is associated to the ring homomorphism:

$$
\iota: \mathbb{Z}[\underline{T}, \underline{D}] \rightarrow \mathbb{Z}\left[M_{2} \times M_{2}\right],
$$

given by $\iota\left(T_{1}\right):=\operatorname{tr}(A), \iota\left(T_{2}\right):=\operatorname{tr}(B), \iota\left(T_{3}\right):=\operatorname{tr}(A B), \iota\left(D_{1}\right):=\operatorname{det}(A)$ and $\iota\left(D_{2}\right):=\operatorname{det}(B)$. The morphism $\tilde{\pi}$ is $\mathbb{G}_{m}$-equivariant and $\mathrm{PGL}_{2}$-invariant, since $\iota$ is homogeneous w.r.t the weights $\operatorname{deg}\left(T_{1}\right)=\operatorname{deg}\left(T_{2}\right)=1$ and $\operatorname{deg}\left(T_{3}\right)=\operatorname{deg}\left(D_{1}\right)=$ $\operatorname{deg}\left(D_{2}\right)=2$, and the Image $(\iota)$ is fixed by the $\mathrm{PGL}_{2}$-action pointwisely.

2.3. The next lemma is easily proven by a use of Euclid division algorithms.

Lemma A. $\mathbb{Z}\left[M_{2} \times M_{2}\right]$ is a free module over $\mathbb{Z}[\underline{T}, \underline{D}]$.

As a consequence of the Lemma $\mathrm{A}, \mathbb{Z}\left[M_{2} \times M_{2}\right]$ is faithfully flat over $\mathbb{Z}[\underline{T}, \underline{D}]$. In particular, the map $\iota(2.2 .2)$ is injective, and we regard $\mathbb{Z}[\underline{T}, \underline{D}]$ as a subring of $\mathbb{Z}\left[M_{2} \times M_{2}\right]$. More strongly, the next lemma says that it is the universal invariant subring with respect to the PGL-action.

Lemma B. Let $R$ be any $\mathbb{Z}[\underline{T}, \underline{D}]$-algebra with 1 . Then

$$
R \simeq\left(R \otimes_{\mathbb{Z}[\underline{T}, \underline{D}]} \mathbb{Z}\left[M_{2} \times M_{2}\right]\right)^{\mathrm{PGL}_{2}} .
$$

Here, the $\mathrm{PGL}_{2}$ action on $\mathbb{Z}\left[M_{2} \times M_{2}\right]$ is extended to the tensor product by letting $\mathrm{PGL}_{2}$ act trivially on $R$.

Remark. (1) It is classically well known that $\mathbb{Q}\left[M_{2} \times M_{2}\right]^{\mathrm{PGL}_{2}}$ is generated by traces $\operatorname{tr}(W)$ for $W \in\{$ the monoid generated by the $A$ and $B\}$ ([G-Y], [Pr1, Pr2]). This is not true for the $\mathbb{Z}$-coefficient. For instance, the relation $2 \operatorname{det}(A)=\operatorname{tr}(A)^{2}-\operatorname{tr}\left(A^{2}\right)$ implies the algebraic dependence of $\operatorname{tr}\left(A^{2}\right)$ and $\operatorname{tr}(A)$ if $\operatorname{char}=2$, whereas $\operatorname{det}(A)$ and $\operatorname{tr}(A)$ are universally algebraically independent as shown in Lemma B.

(2) Donkin $[\mathrm{D}]$ has shown that $\mathbb{Z}\left[M_{n} \times \cdots \times M_{n}\right]^{\mathrm{PGL}_{n}}$ is generated by $\operatorname{tr}(\stackrel{i}{\wedge} W)$ for $i=1, \cdots, n$ and $W \in\left\{\right.$ the monoid generated by $\left.A_{1}, \cdots, A_{m}\right\}$, where we denote by $M_{n} \times \cdots \times M_{n}$ the space of $m$-tuple $n \times n$ matrices $\left(A_{1}, \cdots, A_{m}\right)$.

2.4. For a matrix $A=\left[\begin{array}{ll}a & b \\ c & d\end{array}\right]$, the adjoint matrix $A^{*}$ is the matrix $\left[\begin{array}{cc}d & -b \\ -c & a\end{array}\right]$ so that $A^{*} A=A A^{*}=\operatorname{det}(A) I_{2}$ and $A+A^{*}=\operatorname{tr}(A) I_{2}$.

Definition. The discriminant for $\tilde{\pi}$ is the polynomial in $\mathbb{Z}\left[M_{2} \times M_{2}\right]$ given by:

$$
\begin{aligned}
\Delta(A, B) & :=\operatorname{tr}\left(A B A^{*} B^{*}\right)-\operatorname{tr}\left(A A^{*} B B^{*}\right) \\
& =T_{1}^{2} D_{2}+T_{2}^{2} D_{1}+T_{3}^{2}-T_{1} T_{2} T_{3}-4 D_{1} D_{2} .
\end{aligned}
$$


A justification for this name is given in the next Lemma C. Let $J$ be the ideal of $\mathbb{Z}\left[M_{2} \times M_{2}\right]$ generated by all $5 \times 5$ minors of the Jacobian matrix of $\tilde{\pi}$ :

$$
\frac{\partial\left(T_{1}, T_{2}, T_{3}, D_{1}, D_{2}\right)}{\partial(a, b, c, d, e, f, g, h)}=\left[\begin{array}{cccccccc}
1 & 0 & 0 & 1 & 0 & 0 & 0 & 0 \\
0 & 0 & 0 & 0 & 1 & 0 & 0 & 1 \\
e & g & f & h & a & c & b & d \\
d & -c & -b & a & 0 & 0 & 0 & 0 \\
0 & 0 & 0 & 0 & h & -g & -f & e
\end{array}\right]
$$

Lemma C. The intersection of the Jacobian ideal $J$ with the invariant subring $\mathbb{Z}[\underline{T}, \underline{D}]$ is a principal ideal generated by the discriminant.

$$
J \cap \mathbb{Z}[\underline{T}, \underline{D}]=(\Delta) .
$$

2.5. Let us denote by $D_{\Delta}$ the divisor in $\mathbb{A}^{5}=\operatorname{Spec}(\mathbb{Z}[\underline{T}, \underline{D}])$ defined by the principal ideal $(\Delta)$. Owing to the (2.4.2), the Jacobian criterion implies that the restriction of the morphism $\tilde{\pi}$ (2.2.1) on the complement of $\tilde{\pi}^{-1}\left(D_{\Delta}\right)$

$$
\tilde{\pi}_{\Delta}: M_{2} \times M_{2} \backslash \pi^{-1}\left(D_{\Delta}\right) \rightarrow \mathbb{A}^{5} \backslash D_{\Delta}
$$

is smooth. More strongly, we show in the next lemma, to which the proof of main theorem $\mathrm{A}$ in $\S 4$ is reduced.

Lemma D. The morphism (2.5.1) is a principal $\mathrm{PGL}_{2}$-bundle with respect to the etal topology.

\section{$\S 3 . \quad$ The universal character $\operatorname{ring} R\left(\Gamma, \mathrm{SL}_{2}\right)$}

The universal character ring $R\left(\Gamma, \mathrm{SL}_{2}\right)$ is introduced in this $\S$ by generators and relations in terms of $\Gamma$. The goal of this $\S$ is the formula (3.4.3), which is used in the proof of the main theorem B in $\S 4$ essentially.

\section{1.}

Definition. The universal character ring $R\left(\Gamma, \mathrm{SL}_{2}\right)$ of representations of $\Gamma$ in $\mathrm{SL}_{2}$ is generated by the indeterminates $s(\gamma)$ for $\gamma \in \Gamma$ and divided by the ideal generated by $s(e)-2(e=$ the unit of $\Gamma)$ and by $s(\gamma) s(\delta)-s(\gamma \delta)-s\left(\gamma^{-1} \delta\right)$ for all $\gamma, \delta \in \Gamma$.

$$
R\left(\Gamma, \mathrm{SL}_{2}\right):=\mathbb{Z}[s(\gamma), \gamma \in \Gamma] /\left(s(e)-2, s(\gamma) s(\delta)-s(\gamma \delta)-s\left(\gamma^{-1} \delta\right)\right)
$$

3.2. The ring $R\left(\Gamma, \mathrm{SL}_{2}\right)$ is finitely generated over $\mathbb{Z}$, if $\Gamma$ is finitely generated as shown in the next lemma. Such finiteness was asserted for the ring of traces of $\mathrm{SL}_{2}$ by Fricke [F-K] and proven in [H1, H2, (2.f)], [Ho1, Ho2, Theorem 3.1] and [C-S]. We formulate the finiteness in terms of the universal character ring.

Proposition. Let $A$ be a linearly ordered subset of $\Gamma$, which generates $\Gamma$. Then $R\left(\Gamma, \mathrm{SL}_{2}\right)$ is generated by $G:=\bigcup_{m \in \mathbb{N}}\left\{s\left(\alpha_{1} \cdots \alpha_{m}\right) \mid \alpha_{i} \in A, \alpha_{1}<\cdots<\alpha_{m}\right\}$ over $\mathbb{Z}$.

Corollary 1. If the group $\Gamma$ is finitely generated, then the $R\left(\Gamma, \mathrm{SL}_{2}\right)$ is a finitely generated ring over $\mathbb{Z}$. Hence it is noetherian. 
3.3. For $\alpha$ and $\beta \in \Gamma$, define the discriminant $\Delta(\alpha, \beta) \in R\left(\Gamma, \mathrm{SL}_{2}\right)$ by

$$
\begin{aligned}
\Delta(\alpha, \beta): & =s\left(\alpha \beta \alpha^{-1} \beta^{-1}\right)-2 \\
& =s(\alpha)^{2}+s(\beta)^{2}+s(\alpha \beta)^{2}-s(\alpha) s(\beta) s(\alpha \beta)-4 .
\end{aligned}
$$

This definition of $\Delta$ is parallel to that in (2.4.1). In fact, we shall confuse them in a proof of main theorem $\mathrm{B}$ in $\S 4$. The polynomial has been studied extensively by R. Fricke $[\mathrm{F}]$ and others.

3.4. An importance of the discriminant is explained in the next lemma, which plays an essential role in a proof of the main theorem B in $\S 4$.

Definition. Let $M$ be a $R\left(\Gamma, \mathrm{SL}_{2}\right)$-module. A map $h: \Gamma \rightarrow M$ is called a form with values in $M$, if for any $\gamma$ and $\delta \in \Gamma$ one has a relation:

$$
h(\gamma \delta)+h\left(\gamma^{-1} \delta\right)=s(\gamma) h(\delta)
$$

Lemma. Let $h$ be a form with values in $M$. Suppose $h(e)=h(\alpha)=h(\beta)=h(\alpha \beta)=$ 0 for some $\alpha$ and $\beta \in \Gamma$. Then for any $\gamma \in \Gamma$ one has

$$
\Delta(\alpha, \beta) h(\gamma)=0
$$

Therefore, the images of the values of $h$ by the localization $M \rightarrow M_{\Delta(\alpha, \beta)}$ are zero.

Corollary. For any $\alpha, \beta, \gamma$ and $\delta \in \Gamma$, one has the bilinear expression of $s(\gamma \delta)$

$$
s(\gamma \delta)=-\frac{1}{\Delta(\alpha \beta)}(s(\gamma), s(\gamma \alpha), s(\gamma \beta), s(\gamma \alpha \beta)) \cdot T \cdot{ }^{t}(s(\delta), s(\alpha \delta), s(\beta \delta), s(\alpha \beta \delta))
$$

in the localization $R\left(\Gamma, \mathrm{SL}_{2}\right)_{\Delta(\alpha, \beta)}$, where $T \in M_{4}\left(R\left(\Gamma, \mathrm{SL}_{2}\right)\right)$ is a $4 \times 4$ matrix such that $T \cdot\left(s\left(\xi_{i} \xi_{j}\right)\right)_{i, j=1}^{4}=-\Delta(\alpha, \beta) I_{4}$ for $\xi_{1}=e, \xi_{2}=\alpha, \xi_{3}=\beta$ and $\xi_{4}=\alpha \beta$.

This corollary is the key step to obtain the universality of the character ring, since it implies that the system $(s(\gamma), \gamma \in \Gamma)$ satisfies any algebraic relations which is satisfied by the system $(\operatorname{tr}(\sigma(\gamma)), \gamma \in \Gamma)$ of characters, so far as $\Delta(\alpha, \beta)$ is invertible for some $\alpha, \beta \in \Gamma$.

\section{5 .}

Remark. The study of the algebra of traces (=characters) of representations of a group $\Gamma$ into $\mathrm{SL}_{2}$ started by Voigt and Fricke and is developed by many authors Helling, Horowitz, Magnus, Bass, Lubotzky, Procesi, Platonov and others. See references of the quoted papers.

- Let $F_{n}$ be a free group generated by $n$ elements. Magnus called the homomorphic image of the ring $R\left(F_{n}, \mathrm{SL}_{2}\right)$ in the ring of functions on the representation space $\operatorname{Hom}\left(F_{n}, \mathrm{SL}_{2}(\mathbb{C})\right)$ the ring of Fricke characters $[\mathrm{Ma}]$. We do not know whether the homomorphism has non trivial kernel or not. 


\section{$\S 4$. The invariant morphism $\pi_{\Gamma}$}

In the present $\S 4.1$, we introduce the character variety $\mathrm{Ch}\left(\Gamma, \mathrm{SL}_{2}\right)$, the $\mathrm{PGL}_{2}{ }^{-}$ invariant morphism $\pi_{\Gamma}: \operatorname{Hom}\left(\Gamma, \mathrm{SL}_{2}\right) \rightarrow \mathrm{Ch}\left(\Gamma, \mathrm{SL}_{2}\right)$ and the discriminant $D_{\Gamma}$. The inverse image $\pi_{\Gamma}^{-1}\left(D_{\Gamma}\right)$ consist of the representations $\rho$ such that $\rho(\Gamma)$ is either abelian or reducible ( $\$ 4.2$ Assertion). Then the first main results of the present paper are formulated in 4.3 theorem $\mathrm{A}$ and 4.4 theorem $\mathrm{B}$, which say that the complement $\mathrm{Ch}^{*}\left(\Gamma, \mathrm{SL}_{2}\right):=\mathrm{Ch}\left(\Gamma, \mathrm{SL}_{2}\right) \backslash D_{\Gamma}$ of the discriminant is the regular orbit space of $\mathrm{PGL}_{2^{-}}$ action on absolutely irreducible representations $\operatorname{Hom}^{*}\left(\Gamma, \mathrm{SL}_{2}\right):=\operatorname{Hom}\left(\Gamma, \mathrm{SL}_{2}\right) \backslash$ $\pi^{-1}\left(D_{\Gamma}\right)$

4.1. Let $\Gamma$ be a group. Put

$$
\operatorname{Ch}\left(\Gamma, \mathrm{SL}_{2}\right):=\operatorname{Spec}\left(R\left(\Gamma, \mathrm{SL}_{2}\right)\right)
$$

where $R\left(\Gamma, \mathrm{SL}_{2}\right)$ is defined in $\S 3.1$. Recall the fact that $\operatorname{Hom}\left(\Gamma, \mathrm{SL}_{2}\right)$ is identified with the affine scheme for the universal representation ring $A\left(\Gamma, \mathrm{SL}_{2}\right)$ (cf. $\S 1.3$ Lemma). Then the invariant morphism

$$
\pi_{\Gamma}: \operatorname{Hom}\left(\Gamma, \mathrm{SL}_{2}\right) \rightarrow \mathrm{Ch}\left(\Gamma, \mathrm{SL}_{2}\right)
$$

is defined through the ring homomorphism:

$$
\begin{aligned}
\Phi: \quad R\left(\Gamma, \mathrm{SL}_{2}\right) & \rightarrow A\left(\Gamma, \mathrm{SL}_{2}\right) \\
s(\gamma) & \mapsto \operatorname{tr}(\sigma(\gamma))
\end{aligned}
$$

Comparing relations (3.1.1) with (1.3.1) and (1.3.2), $\Phi$ is well defined. The discriminant subvariety in $\mathrm{Ch}\left(\Gamma, \mathrm{SL}_{2}\right)$ of the morphism $\pi_{\Gamma}$ is defined as

$$
D_{\Gamma}:=\bigcap_{\alpha, \beta \in \Gamma} V(\Delta(\alpha, \beta))
$$

where the discriminant $\Delta(\alpha, \beta) \in R\left(\Gamma, \mathrm{SL}_{2}\right)$ is defined in (3.3.1).

4.2. First, in the next lemma we characterize the inverse image $\pi_{\Gamma}^{-1}\left(D_{\Gamma}\right)$ of the discriminant loci in terms of representation of $\Gamma$.

Assertion. Let $p \in \operatorname{Spec}\left(A\left(\Gamma, \mathrm{SL}_{2}\right)\right)$. Then $p$ belongs to $\pi_{\Gamma}^{-1}\left(D_{\Gamma}\right)$, if and only if the image $\sigma_{p}(\Gamma)$ in $\mathrm{SL}_{2}\left(k_{p}\right)$ of the group $\Gamma$ is either abelian or reducible, where $k_{p}$ is the fraction field of the integral domain $A\left(\Gamma, \mathrm{SL}_{2}\right) / p$ and $\sigma_{p}: \Gamma \rightarrow \mathrm{SL}_{2}\left(k_{p}\right)$ is a representation obtained by a specialization of the universal $\sigma$ at $p$.

4.3. Let us state the first main result of the present paper.

Theorem A. The restriction of the invariant morphism $\pi_{\Gamma}$ to the complement of the inverse image $\pi_{\Gamma}^{-1}\left(D_{\Gamma}\right)$ of the discriminant is a principal $\mathrm{PGL}_{2}$-bundle with respect to the etal topology defined over $\mathbb{Z}$.

Proof. By definition of $D_{\Gamma}$, one has an affine open covering $\mathrm{Ch}\left(\Gamma, \mathrm{SL}_{2}\right) \backslash D_{\Gamma}=\bigcup_{\alpha, \beta \in \Gamma}$ $\operatorname{Spec}\left(R\left(\Gamma, \mathrm{SL}_{2}\right)_{\Delta(\alpha, \beta)}\right)$. Then the proof is reduced to each affine open piece, stated as a consequence of the next Theorem B. 
4.4. For any fixed pair $\alpha$ and $\beta$ of $\Gamma$, consider the $\mathrm{PGL}_{2}$-equivariant morphism $\operatorname{Hom}\left(\Gamma, \mathrm{SL}_{2}\right) \rightarrow M_{2} \times M_{2}$ and a morphism $h_{\alpha \beta}: \operatorname{Ch}\left(\Gamma, \mathrm{SL}_{2}\right) \rightarrow \mathbb{A}^{5}=\operatorname{Spec}(\mathbb{Z}[\underline{T}, \underline{D}])$ defined by the coordinate ring homomorphisms given by

$$
\begin{gathered}
A \mapsto \sigma(\alpha) \text { and } B \mapsto \sigma(\beta), \\
T_{1} \mapsto s(\alpha), T_{2} \mapsto s(\beta), T_{3} \mapsto s(\alpha \beta), D_{1} \mapsto 1 \text { and } D_{2} \mapsto 1
\end{gathered}
$$

Then the next diagram becomes commutative

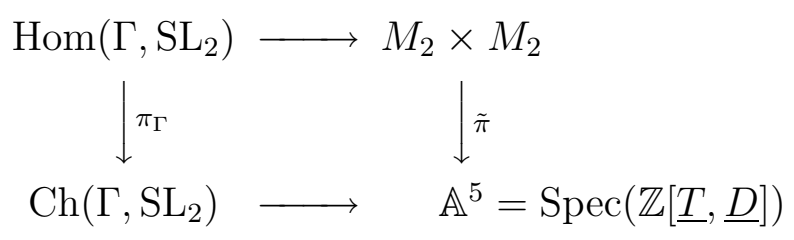

Here we remark that the discriminant $\Delta(\alpha, \beta)$ (3.3.1) is the pull back of $\Delta(A, B)$ (2.4.1). For an abuse of notation, we shall denote both of them by $\Delta$.

Theorem B. The diagram (4.4.3) is Cartesian on the complement of the loci $\Delta=0$. That is: the localization by $\Delta$ of the homomorphism

$$
\Psi_{\alpha, \beta}: R\left(\Gamma, \mathrm{SL}_{2}\right) \otimes_{\mathbb{Z}[\underline{T}, \underline{D}]} \mathbb{Z}\left[M_{2} \times M_{2}\right] \rightarrow A\left(\Gamma, \mathrm{SL}_{2}\right)
$$

(obtained from (4.1.3), (4.4.1) and (4.4.2)) is an isomorphism.

Theorem A follows from the theorem B applied with $\S 2.5$ Lemma D. The theorem B is proved by a construction of the inverse homomorphism for the localization $\left(\Psi_{\alpha, \beta}\right)_{\Delta}$ of (4.4.4) by $\Delta$. This is equivalent to the construction of a representation $\sigma^{*}$ for $a$ prescribed system of "characters" $s(\gamma) \gamma \in \Gamma$ and a pair of matrix $(A, B) \in M_{2} \times M_{2}$ (over the same point of $\mathbb{A}^{5} \backslash\{\Delta=0\}$ ) such that $\operatorname{tr}\left(\sigma^{*}(\gamma)\right)=s(\gamma)$ and $\left(\sigma^{*}(\alpha), \sigma^{*}(\beta)\right)=$ $(A, B)$. Actually, this is achieved by the formula:

$$
\sigma^{*}(\gamma):=-\frac{1}{\Delta(\alpha \beta)}\left(I_{2}, A, B, A B\right) \cdot T \cdot{ }^{t}(s(\gamma), s(\alpha \gamma), s(\beta \gamma), s(\alpha \beta \gamma))
$$

where $T$ is the same matrix in $M_{4}\left(R\left(\Gamma, \mathrm{SL}_{2}\right)\right)$ as in (3.4.3). The multiplicativity $\sigma^{*}(\gamma \delta)=\sigma^{*}(\gamma) \sigma^{*}(\delta)$ is shown by an essential use of the formula (3.4.3).

\section{$\S 5$. Representation variety with real coefficients}

We specialize the result in the previous $\S$ to $\mathbb{R}$-coefficient case. The second main result of the present paper is formulated in 5.5 theorem $\mathrm{C}$, which says that the complement $\mathrm{Ch}\left(\Gamma, \mathrm{SL}_{2}\right)(\mathbb{R})$ of real discriminant $D_{\Gamma}(\mathbb{R})$ decomposes into two semialgebraic sets, which are regular orbit spaces of $\mathrm{PGL}_{2}(\mathbb{R})$ action on $\operatorname{Hom}^{*}\left(\Gamma, \mathrm{SL}_{2}(\mathbb{R})\right)$ and $\mathrm{PU}(2)$ action on $\operatorname{Hom}^{*}\left(\Gamma, \mathrm{SU}_{2}(\mathbb{C})\right)$, respectively. For the purpose we analyze the discriminant $\Delta$ over $\mathbb{R}$ (§5.3 Lemma E). See also [K1, K2, K3, K4], [G1], [H1] and [Ko] for the geometry of the discriminant. 
5.1. Consider the invariant map $\tilde{\pi}$ (2.2.1) over the real number field $\mathbb{R}$.

$$
\tilde{\pi}(\mathbb{R}): M_{2}(\mathbb{R}) \times M_{2}(\mathbb{R}) \rightarrow \mathbb{A}^{5}(\mathbb{R}):=\operatorname{Hom}(\mathbb{Z}[\underline{T}, \underline{D}], \mathbb{R})
$$

given by $\tilde{\pi}(A, B):=(\operatorname{tr}(A), \operatorname{tr}(B), \operatorname{tr}(A B), \operatorname{det}(A), \operatorname{det}(B))$. Consider also the real loci of the discriminant $\Delta(\S 2.4)$.

5.2. Let us introduce an open semialgebraic subset of $\mathbb{A}^{5}(\mathbb{R})$ :

$$
\widetilde{D_{\Delta}}(\mathbb{R}):=\left\{\varphi \in \mathbb{A}^{5}(\mathbb{R}): \Delta(\varphi):=\varphi(\Delta)=0\right\} .
$$

where

$$
\delta_{1}=T_{1}^{2}-4 D_{1}, \delta_{2}=T_{2}^{2}-4 D_{2} \text { and } \delta_{3}=T_{3}^{2}-4 D_{1} D_{2} .
$$

are the discriminants for the characteristic polynomials of $A, B$ and $A B$ respectively. The following facts are shown by direct elementary calculations.

Lemma E. i) $\widetilde{T_{\Delta}}$ is a convex connected component of $\mathbb{A}^{5}(\mathbb{R}) \backslash \widetilde{D_{\Delta}}$. ii) $\mathbb{A}^{5}(\mathbb{R}) \backslash \widetilde{T_{\Delta}}=\tilde{\pi}(\mathbb{R})\left(M_{2}(\mathbb{R}) \times M_{2}(\mathbb{R})\right)$.

Let us decompose the space $\mathbb{A}^{5}(\mathbb{R})$ into semialgebraic sets:

$$
\mathbb{A}^{5}(\mathbb{R})=\widetilde{D_{\Delta}} \amalg \widetilde{H_{\Delta}} \amalg \widetilde{T_{\Delta}},
$$

where $\widetilde{H_{\Delta}}:=\mathbb{A}(\mathbb{R}) \backslash\left(\widetilde{D_{\Delta}} \amalg \widetilde{T_{\Delta}}\right)$ is a union of connected components of $\mathbb{A}(\mathbb{R}) \backslash \widetilde{D_{\Delta}}$. Then the lemma $\mathrm{E} E$ can be paraphrased as: $\operatorname{Image}(\tilde{\pi}(\mathbb{R}))=\widetilde{D_{\Delta}} \amalg \widetilde{H_{\Delta}}$.

5.3. For any given $\alpha, \beta \in \Gamma$, consider the homomorphism $F_{2} \rightarrow \Gamma$ associating the two generators of $F_{2}$ to $\alpha$ and $\beta$. This induces the diagram (4.4.3) over $\mathbb{R}$ :

$$
\begin{array}{ccc}
\operatorname{Hom}\left(\Gamma, \mathrm{SL}_{2}(\mathbb{R})\right) & & \operatorname{Hom}\left(F_{2}, \mathrm{SL}_{2}(\mathbb{R})\right) \subset M_{2}(\mathbb{R}) \times M_{2}(\mathbb{R}) \\
\downarrow \pi_{\Gamma}(\mathbb{R}) & & \downarrow \tilde{\pi}(\mathbb{R}) \\
\operatorname{Ch}\left(\Gamma, \mathrm{SL}_{2}\right)(\mathbb{R}) & \underset{h_{\alpha, \beta}}{\longrightarrow} & \mathbb{A}^{5}(\mathbb{R})
\end{array}
$$

where the morphism $h_{\alpha, \beta}$ is defined by (4.4.2) and $\operatorname{Ch}\left(\Gamma, \mathrm{SL}_{2}\right)(\mathbb{R})=\operatorname{Hom}\left(R\left(\Gamma, \mathrm{SL}_{2}\right)\right.$, $\mathbb{R})$ is the real character variety. The fact that the diagram (5.3.1) is Cartesian outside of the loci $\Delta=0$ ( $\$ 4.4$ Theorem B) together with Lemma E implies the following disjoint decomposition:

$$
\mathrm{Ch}\left(\Gamma, \mathrm{SL}_{2}\right)(\mathbb{R})=D_{\Gamma}(\mathbb{R}) \amalg H_{\Gamma}(\mathbb{R}) \amalg T_{\Gamma}(\mathbb{R}),
$$

where

$$
\begin{aligned}
& D_{\Gamma}(\mathbb{R}):=\left\{t \in \operatorname{Hom}\left(R\left(\Gamma, \mathrm{SL}_{2}\right), \mathbb{R}\right) \mid h_{\alpha, \beta}(t) \in D_{\Delta}(\mathbb{R}) \text { for }{ }^{\forall} \alpha, \beta \in \Gamma\right\} \\
& H_{\Gamma}(\mathbb{R}):=\left\{t \in \operatorname{Hom}\left(R\left(\Gamma, \mathrm{SL}_{2}\right), \mathbb{R}\right) \mid{ }^{\forall} \alpha, \beta \in \Gamma \text { such that } h_{\alpha, \beta}(t) \in \widetilde{H_{\Delta}}\right\} \\
& T_{\Gamma}(\mathbb{R}):=\left\{t \in \operatorname{Hom}\left(R\left(\Gamma, \mathrm{SL}_{2}\right), \mathbb{R}\right) \mid{ }^{\forall} \alpha, \beta \in \Gamma \text { such that } h_{\alpha, \beta}(t) \in \widetilde{T_{\Delta}}\right\} .
\end{aligned}
$$

By definition, $D_{\Gamma}(\mathbb{R})$ is Zariski closed. $H_{\Gamma}(\mathbb{R})$ and $T_{\Gamma}(\mathbb{R})$ are open semialgebraic (Due to the basis theorem of Hilbert, one can find a finite system $\left\{\left(\alpha_{i}, \beta_{i}\right)\right\}_{i \in I}$ such 
that $D_{\Gamma}(\mathbb{R})=\bigcap_{i \in I}\left\{\Delta\left(\alpha_{i}, \beta_{i}\right)=0\right\}$. Then for the same index set $I$, one can show the equalities: $\left.H_{\Gamma}(\mathbb{R})=\bigcup_{i \in I} h_{\alpha_{i} \beta_{i}}^{-1}\left(\widetilde{H_{\Delta}}\right), T_{\Gamma}(\mathbb{R})=\bigcup_{i \in I} h_{\alpha_{i} \beta_{i}}^{-1}\left(\widetilde{T_{\Delta}}\right)\right)$.

5.4. Let us determine the image set of $\pi_{\Gamma}(\mathbb{R})$ as a semialgebraic set (up to the discriminant) in the next lemma. It is proven by a use of the fact that (5.3.1) is Cartesian ( $\$ 4.4$ Theorem B) together with the lemma E.

Lemma F. Let $\pi_{\Gamma}(\mathbb{R})$ be the invariant morphism (4.1.2) defined over $\mathbb{R}$. Then

$$
\operatorname{Image}\left(\pi_{\Gamma}(\mathbb{R})\right) \backslash D_{\Gamma}(\mathbb{R})=H_{\Gamma}(\mathbb{R}) .
$$

A meaning of the set $T_{\Gamma}(\mathbb{R})$ is given by the next lemma. Since $\mathrm{SU}(2) \subset \mathrm{SL}_{2}(\mathbb{C})$, the restriction of $\pi_{\Gamma}(\mathbb{C})$ induces the following map:

$$
\begin{array}{ccc}
u_{\Gamma} \operatorname{Hom}(\Gamma, \mathrm{SU}(2)) & \rightarrow & \mathrm{Ch}\left(\Gamma, \mathrm{SL}_{2}\right)(\mathbb{R}) . \\
\rho & \mapsto s(\gamma) \mapsto \operatorname{tr}(\rho(\gamma))
\end{array}
$$

Lemma G. The image set of the morphism $u_{\Gamma}$ is given by

$$
\operatorname{Image}\left(u_{\Gamma}\right) \backslash D_{\Gamma}(\mathbb{R})=T_{\Gamma}(\mathbb{R}) .
$$

5.5. This is the goal of the present paper. Combining the above (5.4.1), (5.4.2) with the $\S 4$ Theorem A, we obtain principal bundles by the natural adjoint action with respect to the classical topology.

Theorem C. The restrictions of the maps $\pi_{\Gamma}(\mathbb{R})$ (4.1.2) and $u_{\Gamma}$ (5.4.2) to the subset of absolutely irreducible representations $\operatorname{Hom}^{*}\left(\Gamma, \mathrm{SL}_{2}(\mathbb{R})\right):=\operatorname{Hom}\left(\Gamma, \mathrm{SL}_{2}(\mathbb{R})\right) \backslash$ $\pi_{\Gamma}^{-1}\left(D_{\Gamma}(\mathbb{R})\right)$ and $\operatorname{Hom}^{*}(\Gamma, \mathrm{SU}(2)):=\operatorname{Hom}(\Gamma, \mathrm{SU}(2)) \backslash u_{\Gamma}^{-1}\left(D_{\Gamma}(\mathbb{R})\right)$ are a principal $\mathrm{PGL}_{2}(\mathbb{R})$-bundle and a principal $\mathrm{U}(2) / \mathrm{U}(1)$-bundle over the open semialgebraic sets $H_{\Gamma}(\mathbb{R})$ and $T_{\Gamma}(\mathbb{R})$ (5.3.2) of the real character variety $\mathrm{Ch}\left(\Gamma, \mathrm{SL}_{2}\right)(\mathbb{R})$ respectively.

$$
\begin{aligned}
\pi_{\Gamma}(\mathbb{R}): \operatorname{Hom}^{*}\left(\Gamma, \mathrm{SL}_{2}(\mathbb{R})\right) \stackrel{\mathrm{PGL}_{2}(\mathbb{R})}{\longrightarrow} H_{\Gamma}(\mathbb{R}), \\
u_{\Gamma}: \operatorname{Hom}^{*}(\Gamma, \mathrm{SU}(2)) \stackrel{\mathrm{U}(2) / \mathrm{U}(1)}{\longrightarrow} T_{\Gamma}(\mathbb{R}) .
\end{aligned}
$$

The value of a coordinate $s(\gamma) \in R\left(\Gamma, \mathrm{SL}_{2}\right)$ at a point of the base space is equal to the character $\operatorname{tr}(\rho(\gamma))$ for any representation $\rho$ in its fiber.

\section{REFERENCES}

[A] W. Abikoff, The real analytic theory of Teichmüller space, Lect. Notes in Math. 820, Springer, (1980).

[B-L] Hyman Bass and Alexander Lubotzky, Automorphism of groups and of schemes of finite type, Israel J. of Math. 44 No. 1 (1983), 1-22.

[Be] Alan F. Beardon, The geometry of discrete groups, GTM, Springer Verlag, 197.

[Br] G. W. Brumfiel, The real spectrum compactification of Teichmüller space, Contem. Math. 74 (1988), 51-75.

[C-C-G-L-S] D. Cooper, M. Culler, H. Gillet, D. D. Long and P. Shalen, Plane curves associated to character varieties of 3-manifolds, Invent. math. 118 (1994), 47-84.

[C-W] Paula Cohen and Jürgen Wolfart, Modular Embedding for some nonarithmetic Fuchsian groups, preprint, IHES/M/88/57, Nov. 1988.

[C-S] Marc Culler and Peter B. Shalen, Varieties of group representations and splittings of 3manifolds, Annals of Math. 117 (1983), 109-146. 
[D] Stephen Donkin, Invariants of several matrices, Invent. Math. 110 (1992), 389-401.

[F] G. Faltings, Real projective structures on Riemann surfaces, Compos. Math. 48 (1983), 223-369.

[F-K] Robert Fricke and Felix Klein, Vorlesungen uber die Theorie der Automorphen Funktionen, Vol. 1, pp. 365-370, Leipzig: B.G. Teubner 1987, Reprint: New York Johnson Reprint Corporation (Academic Press) 1965.

[G1] William Goldman, Topological components of spaces of representations, Invent. Math. 93 (1988), 557-607.

[G2] William Goldman, Geometric structures on manifolds and varieties of representations, Contemporary Math. 74 (1988), 169-198.

[G-M] F. González-Acuña and José María Montesinos-Amilibia, On the character variety of group representations in $\mathrm{SL}(2, \mathbb{C})$ and $\mathrm{PSL}(2, \mathbb{C})$, Math. Z. 214 (1993), 627-652.

[G-Y] J. H. Grace and A. Young, The ALGEBRA of INVARIANTS, Cambridge Univ. Press., New York, 1903.

[H1] Heinz Helling, Diskrete Untergruppen von $\mathrm{SL}_{2}(\mathbb{R})$, Inventiones math. 17 (1972), 217-229.

[H2] Heinz Helling, Ueber den Raum der kompakten Riemannschen flächen vom geschlecht 2, J. reine angew. Math. 268/269:286-293, (1974).

[H-L-M] M. Hilden, M. Lozano and J. Montesinos-Amilibia, Volumes and Chern-Simons invariant of cyclic coverings over rational knots, These Proceedings, 31-55.

[H-K] C. Hodgson and S. Kerckhoff, Rigidity of hyperbolic cone-manifolds and and hyperbolic Dehn surgery, preprint.

[Ho1] Robert Horowitz, Characters of free groups represented in the two dimensional linear group, Comm. Pure Appl. Math. 25 (1972), 635-649.

[Ho2] Robert Horowitz, Induced automorphisms of Fricke characters of free groups, Trans. Am. Math. Soc. 208 (1975), 41-50.

[J-W] Lisa Jeffrey and Jonathan Weitsman, Bohr-Sommerfeld orbits in the moduli space of flat connections and the Verlinde dimension formula, Comm. Math. Phys. 150 (1992), 593-630.

[J] T. Jørgensen, On discrete groups of Möbius transformations, Amer. J. Math. 98 (1976), 739-749.

[K1] Linda Keen, Intrinsic Moduli, Ann. of Math. 84 (1966), 404-420.

[K2] Linda Keen, On Fricke Moduli, Advances in the theory of Riemann surfaces, Ann. of Math. Studies 66 (1971), 205-2024.

[K3] Linda Keen, A correction to "On Fricke Moduli", Proc. Amer. Math. Soc. 40 (1973), 60-62.

[K4] Linda Keen, A rough fundamental domain for Teichmüller spaces, Bull. Amer. Math. Soc. 83 (1977), 1199-1226.

[Kj] S. Kojima Deformations of hyperbolic 3-cone-manifolds, preprint.

[Ko] Yohei Komori, Semialgebraic description of Teichmüller space, Thesis, Rims, Jan. 1994.

[Kr1] Irvin Kra, Automorphic Forms and Kleinian Groups, W. A. Benjamin Inc., (1972).

[Kr2] Irvin Kra, On ligring of Kleinian groups to $\mathrm{SL}(2, \mathbb{C})$ in Differential Geometry and Complex Analysis, (H. E. Rouch, Memorial Volume)., Springer, Berlin, Heidelberg, New York, 1985, 181-193.

[Kr3] Irvin Kra, Maskit coordinate, Holom. Funct. \& Moduli 2, Math. Sci. Research Inst. Publication 11, Springer, 1992.

[L-M] Alexander Lubotzky and Andy R. Magid, Varieties of representations of finitely generated groups, Memoirs of the AMS, Vol. 58 No. 336, (1985).

[Ma] William Magnus, Rings of Fricke characters and automorphism groups of free groups, Math. Z. 170 (1980), 91-103.

[Mu1] David Mumford, Geometric Invariant Theory, Springer Verlag, Berlin, Heidelberg, 1965, Library of Congress Catalog Card Number 65-16690.

[Mu2] David Mumford, Curves and their Jacobians, (C)y University of Michigan, 1975, All right reserved, ISBN 0-472-66000-4, Library of Congress Catalog Card No. 75-14899.

[N-Z] W. Neumann and D. Zagier, Volumes of hyperbolic 3-manifolds, Topology 24 (1985), 307332.

[O1] Yoshihide Okumura, Fricke moduli and Keen moduli for Fuchsian groups and a certain 
[O2] Yoshihide Okumura, Global real analytic coordinates for Teichmüller spaces, Thesis, Kanazawa Univ., 1989.

[O3] Yoshihide Okumura, On lifting problem of Kleinian group into $\mathrm{SL}(2, \mathbb{C})$, Summer Seminar on Function Theory, July 1992.

[O4] Yoshihide Okumura, On the global real analytic coordinates for Teichmüller spaces, J. Math. Soc. Japan 42 (1990), 91-101.

[P-B-K] V. P. Platonov and V. V. Benyash-Krivets, Characters of representations of finitely generated groups, Proc. of Steklov Inst. of Math. (1991), 203-213.

[Pr1] Claudio Procesi, Finite dimensional representations of algebras, Israel J. of Math. 19 (1974), 169-182.

[Pr2] Claudio Procesi, Invariant theory of $n \times n$ matrices, Adv. Math. 19 (1976), 306-381.

[S1] Kyoji Saito, Moduli Space for Fuchsian Groups, Algebraic Analysis, Vol. II, Academic Press, (1988), 735-787

[S2] Kyoji Saito, The Limit Element in the Configuration Algebra for a Discrete Group, A précis: Proc. Int. Congr. Math., Kyoto 1990, 931-942, Preprint: RIMS-726, Nov. 1990.

[S3] Kyoji Saito, The Teichmüller space and a certain modular function from a view point of group representations, Alg. Geom. and related Topics, Proc. Int. Symp., Inchoen, Republic of Korea, 1992.

[S4] Kyoji Saito, Representation variety of a finitely generated group into $\mathrm{SL}_{2}$ or $\mathrm{GL}_{2}$, Preprint RIMS-958, Dec. 1993.

[S5] Kyoji Saito, Algebraic Representation of the Teichmüller spaces, LMS. Lecture Note Series 200 (1994), 255-288.

[S6] Kyoji Saito, Algebraic Representation of the Teichmüller spaces, Kodai Math. J. 17 (1994), 609-626.

[S-S1] Mika Seppälä and Tuomas Sorvali, Parametrization of Möbius groups acting in a disk, Comment. Math. Helvetici 61 (1986), 149-160.

[S-S2] Mika Seppälä and Tuomas Sorvali, Affine coordinates for Teichmüller spaces, Math. Ann. 284 (1989), 169-176.

[S-S3] Mika Seppälä and Tuomas Sorvali, Trace commutators of Möbius transformations, Math. Scand. 68 (1991), 53-58

[S-S4] Mika Seppälä and Tuomas Sorvali, Geometry of Riemann Surfaces and Teichmüller Spaces, North-Holland Mathematics Studies 169 (1992).

[T] W. Thurston, The geometry and topology of 3-manifolds, Lecture Notes, Princeton Univ., $1977 / 78$.

[V] H. Vogt, Sur les invariants, fondamentaux des équations différentielles linéaires du second ordre, Ann. Sci. Ecole Nrom. sup. 6(3), Suppl.3-72 (1889)(Thèse, Paris).

[W1] Andre Weil, On discrete subgroups of Lie Groups I, Ann. Math. 72 (1960), 369-384,

[W2] Andre Weil, On discrete subgroups of Lie Groups II, Ann. Math. 75 (1962), 578-602,

[We] Jonathan Weitsman, Geometry of the intersection ring of the moduli space of flat connections and the conjectures of Newstead and Witten, preprint 1993.

[Wf] Jürgen Wolfart, Eine arithmetische Eigenschaft automorpher Formen zu gewiisen nichtarithmetischen Gruppen, Math. Ann. 62 (1983), 1-21.

[Wo1] Scott Wolpert, The Fenchel-Nielsen deformation, Ann. of Math. 115 (1982), 501-528.

[Wo2] Scott Wolpert, On the Symplectic Geometry of deformation of a hyperbolic surface, Ann. of Math. 117 (1983), 207-234.

[Wo3] Scott Wolpert, Geodesic length functions and the Nielsen problem, J. Diff. Geom. 25 (1987), 275-296.

[Y] T. Yoshida, On ideal points of deformation curves of hyperbolic 3-manifold with one cusp, Topology 30 (1991), 155-170.

[Z-V-C] Ziechang, Vogt and Colderway, Lect. Notes in Math. 122, 835 and $\mathbf{8 7 5 .}$

RIMS, KYoto UnIVERSITY

E-mail address: saito@kurims.kyoto-u.ac.jp 